\title{
AN EXPERIMENTAL STUDY OF THE SYNOVIAL FLUID OF PATIENTS WITH ARTHRITIS AND SYPHILIS*
}

By ALAN M. CHESNEY, JAROLD E. KEMP, AND FREDERICK H. BAETJER

(From the Departments of Medicine and Roentgenology of the Johns Hopkins Medical School)

(Received for publication, July 6, 1926)

The object of this communication is to present the results of a study of the synovial fluid of a series of patients with arthritis in whom there was serologic or other evidence of the existence of syphilitic infection. The purpose of this study was three-fold: (1) to ascertain if there were any characteristics of the joint fluid of such patients which would be helpful in establishing the diagnosis of syphilitic arthritis, (2) to determine whether or not it is possible to recover Treponema pallidum from the synovial fluid, by rabbit inoculation, in any significant proportion of such cases, and (3) to ascertain if the joints of these patients showed changes visible by the roentgenogram which indicated that the arthritis was syphilitic in origin.

\section{CLINICAL MATERIAL}

Basis of selection. The clinical material for this study comprised a series of ten syphilitic individuals with arthritis who were observed in the Wards or the Out-Patient Department of the Johns Hopkins Hospital, from January, 1924, to January, 1926, inclusive. These patients had not previously received any antisyphilitic treatment according to their own statements. The only qualifications for selection were that the patient must have had clinical or serological evidence of syphilis in addition to his arthritis at the time of examination, that he must have had no antisyphilitic treatment prior to the study, and that there must have been sufficient fluid in the joint cavity to warrant paracentesis and ensure enough material for carrying out all

* Read by title before the American Society for Clinical Investigation, Atlantic City, May 3, 1926. 


\begin{tabular}{|c|c|c|c|c|c|c|}
\hline 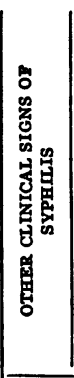 & 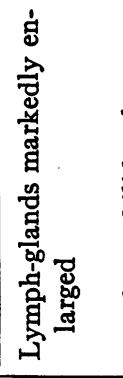 & 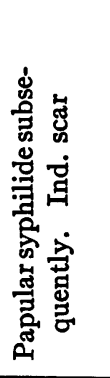 & 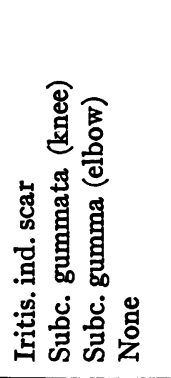 & 号艺 & 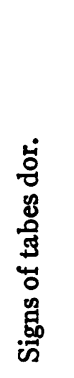 & 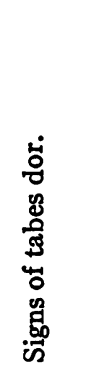 \\
\hline 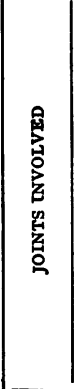 & 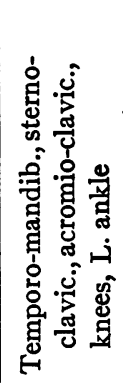 & 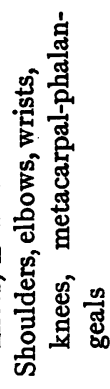 & 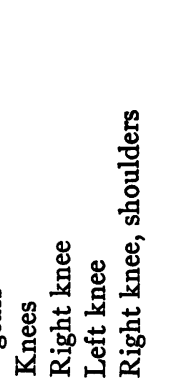 & 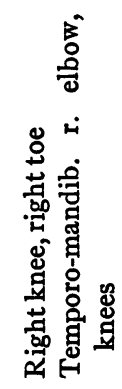 & 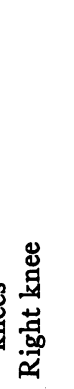 & 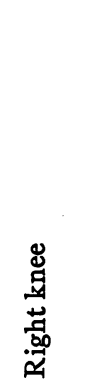 \\
\hline 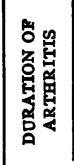 & 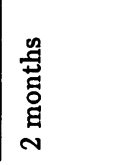 & 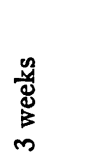 & 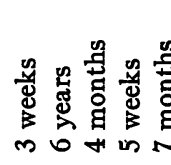 & 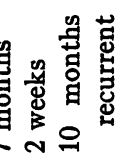 & 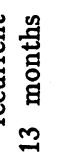 & 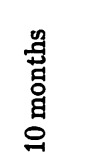 \\
\hline 最景 & 这 & $\sim$ & 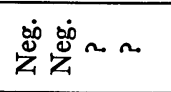 & $\sim \stackrel{800}{a^{\circ}}$ & के & :े \\
\hline 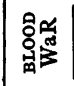 & 最 & 官 & 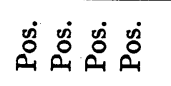 & के & $\stackrel{\infty}{z}^{\circ}$ & में \\
\hline 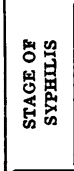 & 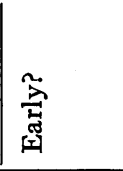 & 育 & 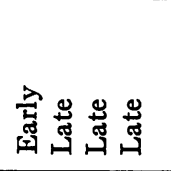 & 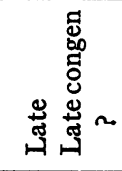 & 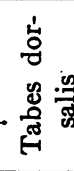 & 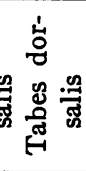 \\
\hline 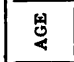 & $\approx$ & $\vec{\sim}$ & นొ & กี ส & 2 & 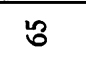 \\
\hline 苗 & Li & $\dot{\Sigma}$ & 芒芒法 & $\dot{\Sigma} \dot{\Sigma}$ & $\dot{\Sigma}$ & $\dot{\Sigma}$ \\
\hline$\$$ & $\dot{\varphi}$ & $\dot{n}$ & 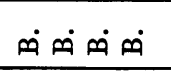 & $\dot{B}$ & $\dot{B}$ & $\ddot{q}$ \\
\hline 界 & $\begin{array}{l}\dot{A} \\
\dot{H}\end{array}$ & $\stackrel{\dot{2}}{\stackrel{1}{\curvearrowleft}}$ & 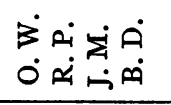 & $\begin{array}{l}\text { 芒 } \\
\dot{2} 0\end{array}$ & $\begin{array}{l}\dot{0} \\
\dot{s}\end{array}$ & نं \\
\hline 完苗 & $\rightarrow$ & $N$ & $m+n$ & $-\infty$ & $a$ & 오 \\
\hline
\end{tabular}


tests. Several patients with arthritis and syphilis came under observation during this period but had to be excluded because there was insufficient fluid to warrant exploratory puncture. The essential clinical data in regard to these cases are presented in Table I, from which it is seen that the patients were for the most part negroes. There were eight males and two females. They were all of adult age.

Duration of syphilis. This information was sought by careful questioning and, in so far as it was possible to judge by the answers of the patient, his age, and the character of the syphilitic lesion present, the stage of the infection has been set down as early or late, it being understood that by "early syphilis" is meant an infection of three years or less, while by "late syphilis" is understood an infection of more than three years duration. We are well aware that such an arbitrary separation is unsatisfactory but it is necessary to set some period for differentiation. Of the ten patients the infection was probably congenital in one and acquired in nine, and of these last it was of recent origin (less than three years) in three and of more remote origin in the remaining six. Two of these six were tabetics.

Character of the arthritis. The arthritis was monarticular in three cases and polyarticular in the remaining seven. In two cases there had been one or more previous attacks of arthritis. The relative involvement of the various joints is shown in table 1. The duration of joint symptoms, exclusive of the patients with tabes, was from two weeks to six years. In the two patients with tabes dorsalis the joints presented all the essential characteristics of Charcot joints. The duration of joint symptoms in these cases was 13 months and 10 months, respectively.

\section{EXPERIMENTAL}

Source of fluid. The fluid in each instance was obtained from the knee joint by paracentesis. If both knees were involved the one most recently showing symptoms was selected. Procaine was used as a local anaesthetic and almost no discomfort attended the procedure.

Examination of fluid. As soon as possible after removal and before clotting, the fluid was subjected to a series of examinations, as follows.

Wassermann reaction. This was performed in the same manner in 
which the routine test is performed in this hospital (1) except that occasionally a joint fluid would be found which was hemolytic, when undiluted, but if diluted 1-4 was no longer such and could be used for the test.

Cytology. Unfortunately the total number of white cells per cu. $\mathrm{mm}$. was not determined in all the cases. Differential counts were made with stained preparations or with fresh preparations examined under the dark field. 'This latter method of examination we have found excellent for differentiating the cells and is convenient in that it affords an opportunity to search for treponemes and to make a differential count at the same time. In reporting our results we have thought it wise to place the lymphocytes and large mononuclear cells in one group and the polymorphonuclears in another.

Bacteriology. Smears. ${ }^{1}$ Smears were stained by Gram's method and examined for bacteria, particularly the gonococcus.

Cultures. ${ }^{1}$ Ordinary aerobic cultures were made on fluid and solid media and in addition cultures were made for the gonococcus on ascitic fluid dextrose agar under partial oxygen tension.

Dark field. All specimens were subjected to dark field examination and at least 100 fields were examined before dismissing the specimen as negative.

Animal inoculation. a. Guinea pig. In each case two guinea pigs were inoculated subcutaneously with 2 to $3 \mathrm{cc}$. fluid and observed for at least ninety days following inoculation. The object of this procedure was to exclude tuberculous infection, if possible.

$b$. Rabbit. Each specimen of fluid was inoculated into the testes of two normal male rabbits. From 2 to $4 \mathrm{cc}$. of fluid was used, depending upon the amount of fluid available and the size of the animal. In most instances both testes were inoculated. Each rabbit was kept under observation for a period of at least ninety days following inoculation.

\section{RESULTS}

The results of the examination of the fluid of these ten patients with arthritis and syphilis are presented in table 2. For the sake of dis-

1 This procedure was carried out in the Bacteriological Laboratory of the Medical Clinic, which is under the supervision of Dr. H. L. Amoss, and we wish to express our gratitude for the coöperation of those serving in that laboratory. 
cussion we have apportioned the cases in three categories, depending upon the amount of evidence to show that the arthritis was actually syphilitic in origin. We have placed the two cases of tabes with Charcot joint in a separate category. The designation of the categories and the basis for apportionment were as follows.

Category I. Cases in which the arthritis was almost certainly syphilitic. In this category we have placed all those patients (and only those) from whose joint fluid treponemes could be recovered. There were three such cases.

Category II. Cases in which the arthritis was very probably syphilitic. In this category we have placed those cases in which there were manifestations of syphilis other than a positive Wassermann reaction and in which antisyphilitic treatment was followed by prompt and marked improvement in the arthritis and at the same time by healing of the other syphilitic lesions. There were two such cases and in both the syphilitic infection was of long duration. In one of these the arthritis had been present for six years, in the other for four months.

Category III. Cases in which there was no reason to believe that the arthritis was syphilitic in origin. In this category we have placed those patients who had no manifestations of syphilis other than a positive Wassermann reaction and in whom there was little or no improvement in the arthritis following antisyphilitic treatment. There were three such patients and in all the duration of the infection was long and in one it was probably congenital in origin. In one of these patients (case 7) Gram positive cocci were demonstrable in smears from the joint fluid and the arthritis only cleared up when an infected mastoid was drained.

Category $I V$. Charcot joints. In this category we have placed the two patients with tabes dorsalis in whom the clinical picture was that of a Charcot joint. In one of these there was no essential change in the joint after intensive antisyphilitic treatment. The other has not been followed for a sufficiently long period, as yet, to determine the effect of treatment upon the arthritis.

Analysis of table 2 brings out several interesting facts. In the first place it is seen that by rabbit inoculation virulent treponemes were recovered from the joint fluid in three of the ten cases. In all of these the syphilis was recent in origin, and in fact they were the only pa- 


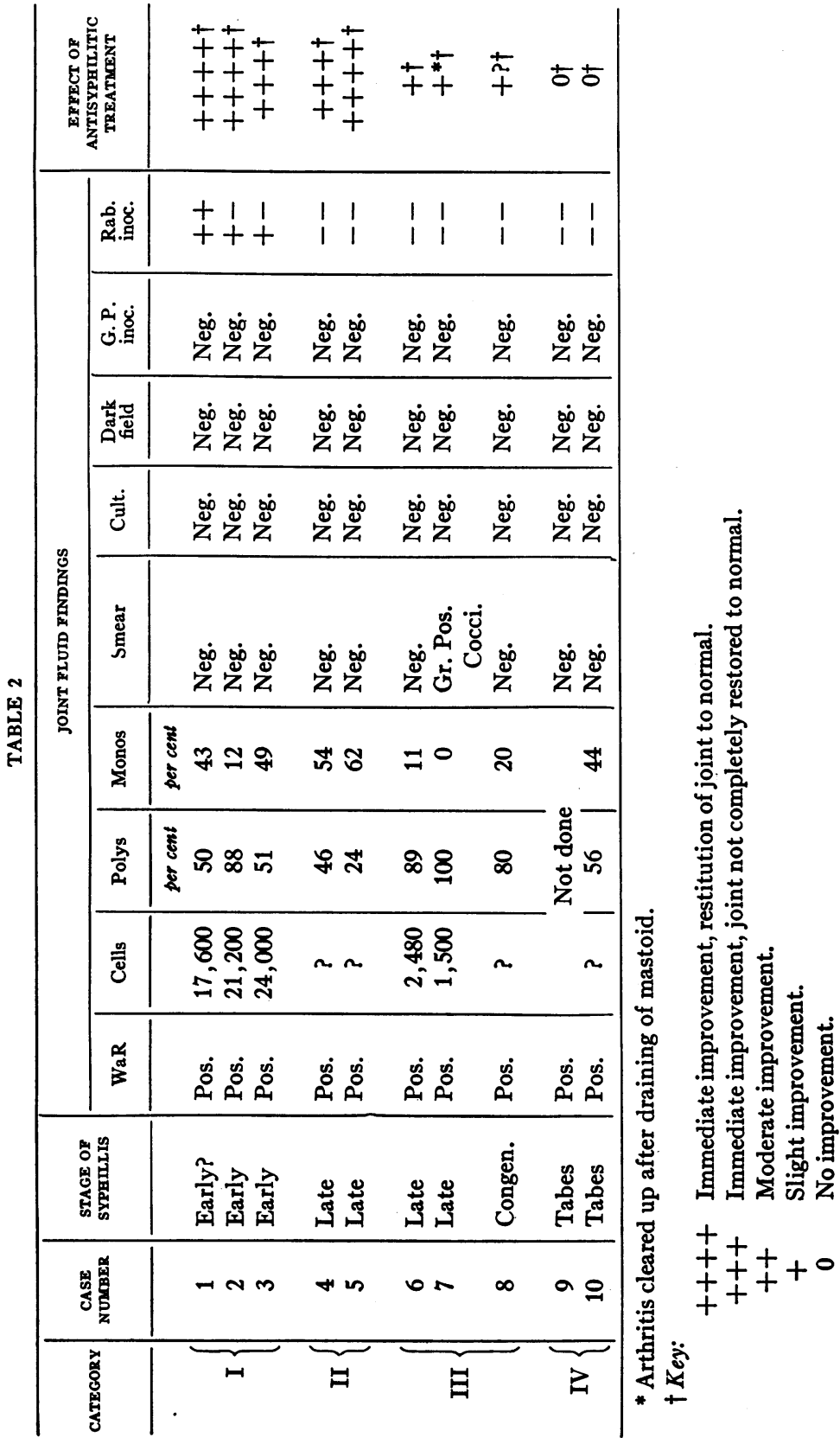


tients with early syphilis and arthritis whom we had an opportunity to study in the manner indicated. They will be considered in greater detail below. Treponemes were not recovered from the joint fluid in any other instance. Dark field examination of the fluid was negative for treponemes in every instance.

Positive Wassermann reactions were obtained with the joint fluid in every instance in which the blood Wassermann reaction was positive. This was to be expected, of course. In one instance (case 9, tabes with Charcot joint) the Wassermann reaction of the joint fluid was positive and that of the blood negative.

Considering the cases in categories I and II as being instances of true syphilitic arthritis, and hence capable of being considered as a group, we find that in four out of five there was a marked tendency of the joint fluid to show a relatively high percentage of lymphocytes and mononuclears, taken together ( 43 to 62 per cent). The percentage of these cellular elements in the synovial fluid of these cases was, as a rule, higher than that of the corresponding cells in the blood. In case 2 , in which the proportion of lymphocytes and mononuclears together in the synovial fluid did not exceed 12 per cent, there is some reason to believe that the arthritis may have been due, in part at least, to an antecedent gonococcal urethritis, although no gonococci were recovered from the joint fluid.

Of the diagnostic significance of this finding of relatively high percentage of lymphocytes and mononuclear cells in the synovial fluid we are as yet uncertain. The cases are too few to permit of generalisation on this point, but it is of interest to note that the same tendency was not observed in those cases in which the therapeutic response to antisyphilitic treatment gave no reason to believe that the arthritis was syphilitic in origin. Swift (2) has found a high percentage of polymorphonuclear cells in the joint fluid in cases of acute rheumatic fever, and Labor and Von Balogh (3) found the polymorphonuclear cells predominant in the joint fluid in cases of acute rheumatic fever, gonococcal arthritis and arthritis developing during the course of dysentery, although in the latter group the lymphocytes sometimes were predominant when the inflammatory process was clearing up. Singer (4) found polymorphonuclears predominating in the joint fluid of patients with dysentery and arthritis. Griffon and Abrami (5) 
studied the joint fluid obtained from a patient with recurrent secondary syphilis and coincident arthritis which yielded rapidly to mercurial treatment but relapsed following cessation of treatment. The fluid at first showed what the authors called a "polynuclear formula" which later became lymphocytic as the process became sub-acute. Unfortunately no further details of the cytology are given.

There are comparatively few statements in the literature as to the cytology of the joint fluid in cases of syphilitic arthritis and it is not beyond the range of possibility that the relatively high percentage of lymphocytes and mononuclear cells, such as we encountered in the presumably syphilitic joints, may prove to be a constant finding in syphilitic arthritis and help to differentiate it from other types of acute and subacute polyarthritis. Our cases are, of course, too few to warrant any conclusions as yet but they suggest that the point is worthy of further attention.

\section{CLINICAL FEATURES}

It has seemed desirable, for purposes of record, to present in some detail the clinical histories of the patients in whom there was good evidence for believing that the arthritis was syphilitic in origin, that is to say, those falling in categories I and II.

\section{Category I. Joint fluid inoculation positive}

Case 1. L. D., Service No. 50596. The history of this case has already been published in detail (6) and will be but briefly summarised here. The patient was a. colored woman 23 years old. She had been married 4 years and her husband was known to have had syphilis. She had never had arthritis previously. Two months prior to admission she began rather suddenly to have stiffness and pain in the neck, shoulders and knees, and she was obliged to go to bed shortly afterwards. A month later there was sore throat followed by swelling of the glands in the neck.

On examination she was found to have marked swelling of all the superficial lymph nodes of the body which in addition were quite painful. There was pain, tenderness and limitation of motion of the temporo-mandibular, sterno-clavicular, acromio-clavicular, knee and left ankle joints. No joint could be said to be exquisitely tender. Both knees were moderately swollen and fluid was present in each. The liver and spleen were palpable. The blood Wassermann was positive and the blood count revealed an eosinophilia of 10 per cent. The roentgen-ray examination was reported as follows: (130779) "Examination of the left knee 
shows slightly increased amount of fluid in the joint, indicated by riding of the patella; no bone changes or any evidence of involvement of cartilage; no evidence of any periostitis just adjacent to the joint. From an X-ray standpoint, with the exception of moderate hydrops, the examination is negative." There was slight irregular elevation of temperature, the maximum attained being $100.5^{\circ} \mathrm{F}$.

Rest in bed was not followed by any marked improvement but following the administration of neo-arsphenamine and arsphenamine there was a prompt improvement in the joint symptoms and diminution in size of the lymph nodes. The arthritis disappeared entirely within 3 weeks after the initiation of antisyphilitic treatment, leaving entirely normal joints. A lymph node was excised prior to treatment and inoculated into two rabbits. In both of these animals syphilitic orchitis developed. As indicated in table 2, the joint fluid also gave a positive result on inoculation. The patient has been under constant supervision for a period of over two years and there has never been any recurrence of the arthritis.

Comment. The case is of particular interest because there were no cutaneous manifestations of syphilis at any time, yet the infection was in all probability fairly recent. The patient was regarded, at first, as having an acute polyarthritis of unknown etiology until the positive Wassermann reaction directed attention to the possibility of syphilis as the cause.

Case 2. J. S., Service No. 52059. The patient, a colored male, 21 years of age, had never had arthritis prior to the present attack. He had had an acute gonococcal urethritis shortly before the onset of the present trouble. Three weeks prior to admission he began suddenly to have pain in both shoulders followed almost immediately by painful swelling of both knees.

On examination the shoulders, elbows, wrists, knees and metacarpal-phalangeal joints were painful on motion and tender on palpation. The right shoulder, both elbows and both knees were most involved, with fluid in the latter, but no joint was exquisitely tender. There was moderate general lymph node enlargement and a bilateral conjunctivitis. There was no urethral discharge but an indurated area could be palpated on the shaft of the penis.! Leucocytes, which were 9,100 on admission, reached a maximum of 16,000 within a week and the temperature rose as high as $101^{\circ}$. The differential count was normal. The blood Wassermann reaction was positive. The roentgen-ray examination was reported as follows: (146592) "Examination of the right knee shows an increase in the synovial fluid; indicated by riding of the patella. No changes in the synovial membranes or bones; no evidence of any periostitis. The joint is negative, except for hydrops."

The joint fluid, aspirated 4 days after admission, gave a positive inoculation result in one of two rabbits. There was some improvement under rest in bed and salicylates but certainly not marked. Sixteen days after admission a maculopapular syphilide made its appearance. Following the administration of ars- 
phenamine the rash quickly disappeared as well as the arthritic symptoms, and the joints were quickly restored to normal. The patient subsequently stopped coming for treatment and following this lapse a neuro-recurrence developed.

Comment. This case is of considerable interest from a diagnostic standpoint because of the possibility of a recent gonococcal infection being involved in the causation of the arthritis. This possibility must be kept in mind, although gonococci were not demonstrable in the conjunctival secretion nor in the joint fluid, whereas treponemes were recovered from the latter. It is of interest that the onset of joint symptoms preceded the occurrence of the syphilitic rash by about five weeks. Wile (7) has reported a case of early syphilitic arthritis in which the joint symptoms preceded the appearance of cutaneous lesions by at least two months.

Case 3. O. W., Service No. 53251. The patient, a colored male, 55 years of age, unmarried, had had "rheumatism" in the right knee for about a year previous to admission. It was not sufficient to keep him from his work which was that of a fireman on a vessel. Two months and a half prior to admission he acquired pneumonia from which he recovered. While convalescent from this attack and while yet in bed a sore appeared on the penis. (Two weeks prior to the onset of the pneumonia he had been exposed to venereal infection.) Several days after the appearance of the penile sore the glands in the left groin began to swell, attained the size of a hen's egg and then receded somewhat, without suppuration. This was followed by pain and redness in the eyes, photophobia and lacrimation. Three weeks before admission the left knee became painful, hot, and somewhat swollen, then the left ankle and right knee became involved but were not as painful as the left knee.

On examination there was found a bilateral conjunctivitis and iritis, involving the right eye more than the left. The inguinal glands were markedly enlarged and hard, especially on the left, and there was an indurated scar on the prepuce which had all the characteristics of a healed chancre. There was a moderate genu valgum due to deformity of the right knee which was swollen. This swelling involved the lower end of the femur and the soft parts as well. There was crepitus on motion, a small amount of fluid in the joint, slight atrophy of the muscles of the thigh and slight limitation of motion. The left knee was swollen and contained a moderate amount of fluid. It was tender and painful on motion but not exquisitely so. No bone changes could be made out and there was no limitation of motion. All other joints were normal. The roentgen-ray examination was reported as follows: (156993) "Examination of the left knee shows slight swelling of the synovial membranes. There is apparently no increase in fluid, as the patella is not riding. There are slight spur-like exostoses arising from ends of patella and external edge of left tibial articulation. 
"Examination of right knee shows marked swelling of the synovial membranes, marked arthritis of joint, indicated by some destruction of cartilage of femur with formation of fairly marked exostoses from the under surface of patella, and similar but slight exostoses arising from edges of the articulating surface of tibia; also slight periostitis of femur just near where the synovial membrane is attached. This patient's age is $\mathbf{5 5}$ years, and the changes seen in the joint itself are those of a hypertrophic arthritis. The only change suggestive of luetic infection is slight periostitis of femur outside the joint." The blood count showed 8760 cells per cubic millimeter with 7 per cent eosinophiles and 53 per cent neutrophiles. The maximum temperature attained was $100.0^{\circ} \mathrm{F}$. A drop of fluid was aspirated from a left inguinal lymph node and after prolonged search with the dark field microscrope a single treponeme was found. There was no acute urethritis.

Following antisyphilitic treatment there was a subsidence of the iritis and conjunctivitis, and marked improvement in the condition of the knees, although complete restoration to normal was not attained, the right knee still showing changes but very little fluid. Upon discharge from the hospital the patient disappeared from observation.

Comment. This case must be regarded as one in which there was an antecedent chronic arthritis of moderate degree, more marked on the right than the left, that exhibited an acute exacerbation following syphilitic infection. The changes in the joint as revealed by clinical and roentgenological examination were essentially those of a long standing process involving bone, cartilage and synovial membrane, upon which an acute hydrops had been superimposed. There seems little doubt but that syphilitic infection played a prominent rôle in the exacerbation of the joint symptoms but by no stretch of the imagination can it be conceived of as having been responsible for the changes in the bone and cartilage, which must have occurred long before the patient acquired his syphilis. It is quite conceivable, however, that the pre-existing chronic arthritis acted to create a favorable nidus for the localisation of treponemes in the joint structures with consequent initiation of a syphilitic process, since experimental evidence has been forthcoming to show that an area of inflammatory reaction in the subcutaneous tissues of the rabbit constitutes a favorable place for the inoculation of treponemes (8).

Summary. In these three cases we are dealing with patients in the early period of syphilitic infection, exhibiting a polyarthritis of moderate severity with slight fever, from whose synovial fluid characteristic and virulent examples of $T$. pallidum could be recovered by 
rabbit inoculation. In one, the clinical picture was that of an acute process superimposed upon an old (non-syphilitic) arthritis, in the others the acute process involved joints hitherto unaffected. There were no significant features, in connection with the joint changes, from either the clinical or the roentgenological point of view, which would serve to distinguish the arthritis presented by these patients from other types of arthritis of the same degree of severity. One striking feature was the relatively slight degree of pain and tenderness of the joints and the absence of high fever. The polyarthritis in all three patients yielded rapidly to antisyphilitic treatment.

\section{Category II. Marked improvement under antisyphilitic treatment}

The two cases which we have placed in Category II are similar in that they both were examples of monarticular arthritis developing late in the course of syphilis, accompanied by cutaneous gummata and yielding promptly to arsphenamine therapy. They fall into that group of cases of syphilitic arthritis, monarticular in distribution, that have a definite resemblance to tuberculous arthritis, and that have occupied the attention of French writers particularly $(9,10)$.

Case 4. R. P., Service No. G-83694. The patient, a colored male, 38 years of age, had had a single penile lesion 10 years prior to admission and 4 years prior to the onset of the present illness. This lesion was self-treated and healed, leaving a scar. It was not followed by any of the usual manifestations of early syphilis. Six years before admission the right knee joint became swollen and stiff and remained so thereafter. It was red and somewhat tender at times, more so about 10 weeks before admission when hard lumps appeared in the region of the knee beneath the skin. Four weeks after their appearance these masses softened and discharged their contents by way of sinuses. Four years previously there had been a swelling of the left clavicle which had broken through the skin and discharged for a while but healed spontaneously.

Examination showed an extensive scar on the shaft of the penis, thickening of the middle portion of the left clavicle with a scar over this area, and a markedly swollen right knee. This swelling involved the periarticular and the subcutaneous tissue, especially above the knee, and there was marked riding of the patella. There was grating on passive motion of the right knee, which flexed to $90^{\circ}$ only, but the joint was not hot nor very tender. Above the knee there were several large communicating sinuses in the midst of an area of infiltrated tissue. There was considerable ulceration of the skin where these sinuses issued. The 
general appearance was that of typical subcutaneous gummata which had broken through the skin. (See fig. 1.) No other joints were involved. The roentgenray examination was reported as follows: (121756) "Examination of the knee does not show any increased fluid in the joint. Some swelling around joint-probably

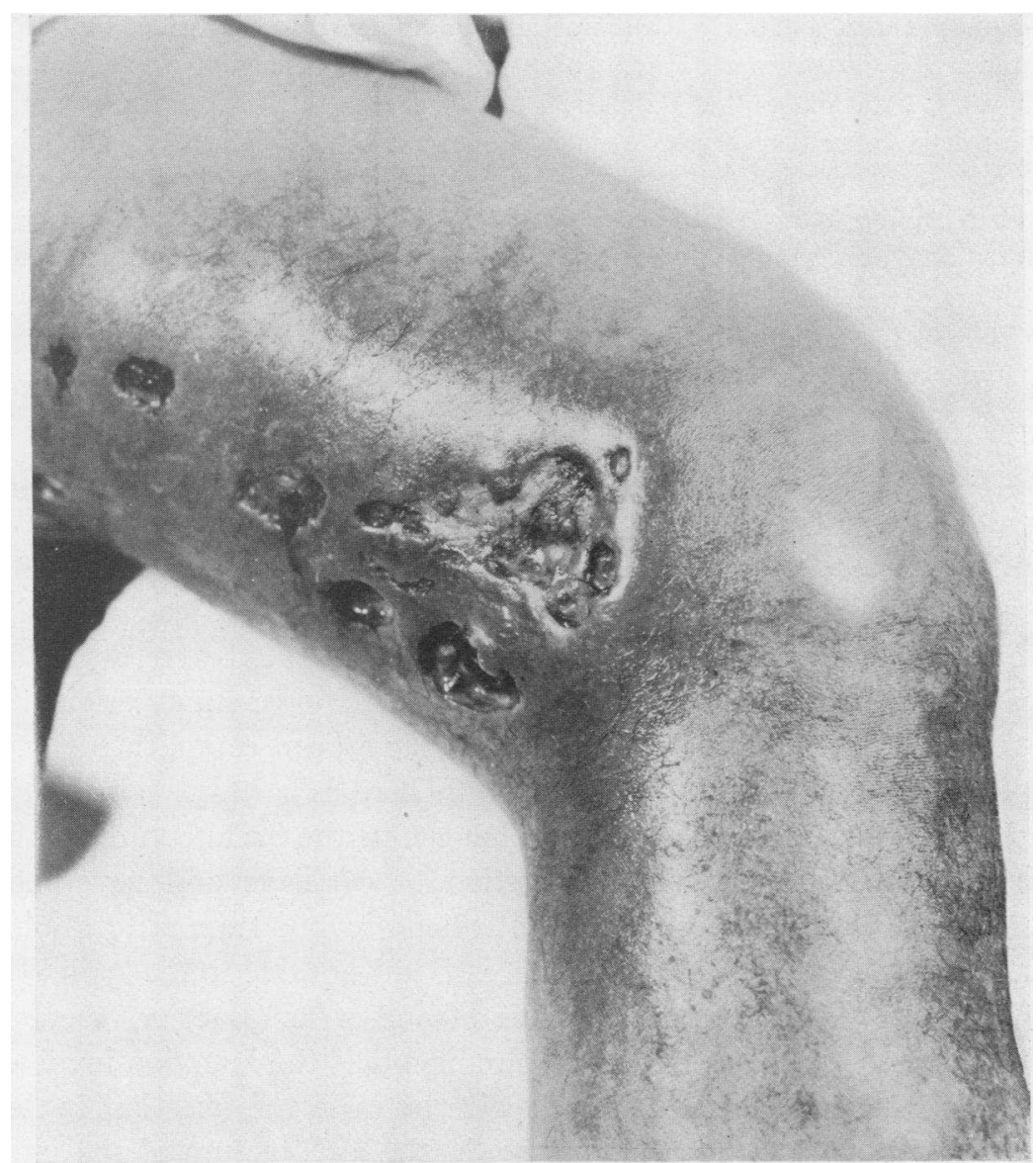

Fig. 1. Right Knee of Case 4, R. P., Before Treatment, Showing Swelling of Joint and Subcutaneous Gummata

tissues external to the synovial membrane. There is, however, some destruction of cartilage and formation of new bone on the top surface of the femur, and beginning spur formation along the articulating edge of the tibia. No evidence of any periostitis. The changes impress one as being non-syphilitic in character, but are those of an infectious arthritis." Fluid from the knee joint yielded negative 
results on animal inoculation. It showed 46 per cent polymorphonuclear cells and 54 per cent lymphocytes and mononuclear cells combined.

Under expectant treatment there was no improvement but following the institution of antisyphilitic therapy there was prompt and marked improvement in the knee. The sinuses healed, the swelling largely subsided, pain and tenderness disappeared, and the patient was able to resume his occupation, that of a waiter. This improvement was manifest after two injections of arsphenamine, and was so satisfactory that the patient no longer thought it necessary to return for further treatment and was persuaded to do so only with difficulty. The joint was not completely restored to normal, even after intensive antisyphilitic treatment extending over a period of a year, but the residual alterations were not marked and from the functional standpoint the result was entirely satisfactory.

Comment. There can be little doubt from the prompt and remarkable response to antisyphilitic treatment, " that syphilitic infection played an important part in the pathogenesis of the arthritis in this patient. Whether or not it is solely responsible for the changes in the architecture of the joint may perhaps be debatable. The possibility should be entertained that the changes may in part have been due to secondary infection, since there were gummatous sinuses leading to the periarticular tissue, but the cultures of the joint fluid were negative. Again there is the possibility that the arthritic process may originally have been due to some other cause and have been complicated by a superimposed late syphilitic process. There is a third possibility, namely that the rather extensive changes in the architecture of the joint, as demonstrated by destruction of cartilage and new bone formation, may have been the result of continuous trauma to a joint that was the seat of a syphilitic process, and not due to the syphilitic infection per se. In the experience of one of us (Baetjer) there is little evidence to show that in acquired syphilis the infection involves the cartilage, hence it has seemed wise to raise the question, in this particular case, whether the changes in the architecture of the joint and more particularly the destruction of cartilage is to be attributed to the syphilitic inflammatory processes, or whether some other factor may not also have played a part. These are matters of speculation and cannot be resolved now so far as this particular case is concerned, but the fact remains that antisyphilitic treatment in this patient brought about a really remarkable improvement within a very short 
space of time and converted an incapacitating joint into one which functioned almost normally.

Case 5. J. M., Service No. G-97672. The patient, a colored male, aged 28, had had a penile lesion accompanied by inguinal adenitis 8 years previous to admission. The sore was cauterised by a physician and healed in two weeks without any subsequent secondary manifestations. His wife had one miscarriage, no other pregnancies. Four months prior to admission he sustained an injury to the right elbow and left knee. Both joints became swollen and painful and shortly afterward two or three small ulcers appeared in the region of the elbow. The condition remained practically unchanged during the succeeding 4 months.

On admission there was found an indurated area $8 \times 5 \mathrm{~cm}$. on the flexor surface of the right elbow, with a punched out ulcer $2 \mathrm{~cm}$. in diameter in the center and several smaller ulcers and pin-hole openings in the remainder of the lesion, all of which exuded a sticky exudate. There was no tenderness of the ulna and motion of the elbow was unrestricted. It was thought that the joint itself was not involved in the process, which was regarded as a diffuse gummatous lesion. There was swelling of the left knee and of the left pre-patellar bursa with evidence of fluid in the joint; the latter, however, was not tender nor hot. Flexion was limited by about $15^{\circ}$ and there was no crepitus. The roentgen-ray examination was reported as follows: (134483) "Examination of the knee does not show any fluid in the joint and no changes in the cartilage or bones. No evidence of any periostitis. From an X-ray standpoint the examination is negative." The fluid obtained from the knee gave entirely negative bacteriological results, both on culture and animal inoculation. It contained 4800 cells per cubic millimeter of which 24 per cent were polymorphonuclears and 62 per cent were lymphocytes and mononuclears. The remaining 14 per cent were unclassified.

Following the administration of arsphenamine there was prompt healing of the gumma of the elbow and an equally prompt return of the knee joint to normal. The improvement was maintained.

Comment. The case is of interest in that there was a clear history of trauma preceding the onset of the arthritis. Local treatment had not brought about any improvement in the condition within a period of four months but the response to antisyphilitic treatment was prompt and indeed marked. The arthritis of the knee cleared up as rapidly as did the gummatous process in the neighborhood of the elbow.

Summary. In both of the foregoing cases the arthritis was monarticular, involved the knee, and the swelling of the joint was rather out of proportion to the other signs of inflammation. Local heat and tenderness were present in both to a relatively slight extent, so that 
the disease of the joint resembled in some respects tuberculosis. In both the response to arsphenamine was striking. From the clinical standpoint these two cases present a striking contrast to those in the foregoing category, yet the improvement of the joint under antisyphilitic treatment was so prompt and so marked, and paralleled so closely the healing of the gummata that there can be little doubt but that syphilitic infection played a rôle in the pathogenesis of the arthritis. These cases correspond more or less closely with the picture described years ago by Richet (11). He drew attention to a form of monarticular arthritis occurring in a syphilitic individual late in the course of the disease, characterised by considerable swelling of the joint with relatively slight pain, tenderness and elevation of temperature of the joint itself, and yielding readily to antisyphilitic treatment.

\section{Category III. Probably non-syphilitic}

In the cases falling in Category III, there was nothing in the response to arsphenamine treatment to suggest that syphilis was responsible for the arthritis, and animal inoculation was negative. Indeed in one (No. 7), Gram positive cocci were seen in the smear made from the joint fluid and in this case the arthritis subsided promptly when an infected mastoid was drained. None of the cases in this group are deemed worthy of extended comment.

\section{Category IV. Charcot joint}

Both of the cases of tabes with Charcot joint yielded negative joint fluids from the bacteriological standpoint; nor was there any immediate significant change in the condition of the joint itself following antisyphilitic treatment. The histories are not of sufficient interest to warrant further details.

\section{ROENTGENOLOGICAL FINDINGS}

Study of the roentgenograms in the five cases (Categories I and II) which we have regarded as examples of syphilitic arthritis has not revealed any new principle which can be utilised in the roentgenological diagnosis of syphilitic arthritis. The $\mathrm{X}$-ray did not disclose any change in the joints in these cases which was common to all and could 
be regarded as peculiarly syphilitic. Such changes as were observed were largely those associated with increase in the fluid content of the joint. It is true that in one of the cases bony changes were found but the history of the case showed clearly that these were associated with a pre-existing hypertrophic arthritis of the sort commonly seen in elderly persons.

Failure of these cases to show any roentgenological signs indicative of syphilis is not, however, to be taken as evidence that the roentgen ray may not disclose syphilitic changes in a diseased joint. The X-ray can and does at times reveal changes in joints which strongly suggest the presence of syphilis as a determining factor in the production of the joint abnormality. This is true in acquired syphilis as well as in the inherited type of the disease. It has been the experience of one of us (Baetjer) that in adults hydrops of the knee joint associated with periostitis immediately adjacent to the joint, where there is no history of trauma, is strongly suggestive of syphilis. Moreover in the Charcot joints that develop in patients with syphilitic disease of the central nervous system the joint changes as revealed by the $\mathrm{X}$-ray are pathognomonic of this condition. In these two groups of cases, then, namely those with Charcot joints and those in which there is periostitis associated with hydrops, the X-ray may be expected to be of value in diagnosis. In addition to these two groups, however, there is still a third and probably fairly large group of cases showing hydrops only, without periostitis, and in this group the $\mathrm{X}$-ray does not reveal any changes which may be looked upon as syphilitic in origin. It so happened that all five of our cases which were regarded as examples of syphilitic arthritis fell within this group. What proportion of cases of syphilitic arthritis in general will be found to fall within this group it is impossible to say, but it seems to us essential that the limitations of the roentgenologic method in the diagnosis of syphilitic arthritis be kept clearly in mind.

\section{SUMMARY}

Ten cases of arthritis ocurring in patients with clinical or serologic evidence of syphilis have been studied with reference to (a) abnormalities of the synovial fluid, (b) presence of treponemes or other microorganisms in the synovial fluid as determined by rabbit and guinea 
pig inoculation and by culture, (c) response to antisyphilitic treatment and (d) roentgenologic findings. The cases represented various stages in the course of the infection, including two patients with tabes dorsalis and Charcot joints. From the response to treatment the arthritis was regarded as syphilitic in origin in 5, or one half of the cases. From the synovial fluid of 3 of these 5 patients strains of Treponema pallidum virulent for rabbits were obtained by inoculation of animals of that species. In 4 of these 5 the synovial fluid showed a relatively high percentage of lymphocytes and mononuclear cells combined. No roentgenological findings significant of syphilis were encountered in these patients. The clinical findings are reported in detail.

\section{BIBLIOGRAPHY}

1. Keidel, A., and Moore, J. E.: Bull. Johns Hopkins Hosp., 1923, xxxiv, 16. The Wassermann Reaction in the Johns Hopkins Hospital.

2. Swift, H. F.: Personal communication.

3. Labor, M., and Von Balogh, E.: Wien. Klin. Wochnschr., 1919, xxxii, 535. Zytologische und Serologische Untersuchungen der Synovia im Besonderen bei Akuten Gelenksentzundungen.

4. Singer, G.: Münch. Med. Wochnschr., 1915, lxii, 183. Erfahrungen aus der letzten Dysenterieepidemie.

5. Griffon, V., and Abrami, P.: Cited in Annales des Mal. Vener., 1907, ii, 55 L'hydarthrose de la Syphilis Secondaire. Etude Cytologique et expérimentale de l'épanchment.

6. Chesney, A. M., Kemp, J. E., and Resnik, W. H.: Bull. Johns Hopkins Hosp., 1924, xxxv, 235. Syphilitic Arthritis with Eosinophilia Recovery of T. pallidum from the Synovial Fluid.

7. Wile, U. J.: Jour. Cut. Dis., 1914, xxxii, 20. Arthropathy in Secondary Syphilis.

8. Chesney, A. M., and Kemp, J. E.: Jour. Exp. Med., 1925, xli, 487. Studies in Experimental Syphilis. II. The influence of a Non-Specific Inflammatory Reaction Upon the Development of the Chancre.

9. Pillon, M.: Les Formes Cliniques de la Syphilis Articulaire. Thèse, Lyon, 1914.

10. Gouariantz, R.: Contribution a l'Etude des Arthropathies Syphilitiques. Thèse, Lyon, 1914.

11. Richet: Mem. de l'Acad. de Méd., 1853, xvii, 249. 